\title{
On the geometry of warped spacetimes
}

\author{
J Carot $\dagger$ and J da Costa \\ † Departamento Física, Universidad Illes Balears, E-07071 Palma de Mallorca, Spain \\ $\ddagger$ Departamento Matemática, Universidad da Madeira, Funchal, Portugal
}

Received 3 August 1992

\begin{abstract}
An invariant characterization of warped spacetimes is given and a classification scheme for them is proposed. Some results on the curvature structure (Petrov and Segre types of the Weyl and Ricci tensors) are given and a thorough study of the isometry group that each class of warped spacetime may admit is carried out.
\end{abstract}

\section{Introduction}

Given two manifolds (one Lorentzian and one Riemannian) $\left(M_{1}, h_{1}\right)$ and $\left(M_{2}, h_{2}\right)$ and given a smooth function $\theta: M_{1} \rightarrow \Re$ (henceforth called the 'warping function'), one can build a new Lorentz manifold $(M, g)$ by setting $M=M_{1} \times M_{2}$ and $g=h_{1} \oplus e^{2 \theta} h_{2}$.

We call $(M, g)$ a 'warped product manifold' and denote it as $M \equiv M_{1} \times_{\theta} M_{2}$. The case $\operatorname{dim} M=4$ corresponds to $(M, g)$ being a spacetime, and will therefore be called a 'warped product spacetime' (or simply 'warped spacetime') from now on.

The aim of this paper is to study these warped spacetimes from both a geometrical and a physical point of view. It should be noted that the study of such spacetimes is of interest in general relativity, since they comprise a wide variety of exact solutions to Einstein's field equations: Bertotti-Robinson, Robertson-Walker, Schwarzschild, Reissner-Nordstrom, de Sitter, etc. Also warped spacetimes can be regarded, in some sense, as generalizations of locally decomposable spacetimes in the sense usually meant in general relativity $[1,2]$.

Special types of warped spacetimes have been studied by Allison $[3,4]$, Beem and Powell [5], Beem and Ehrlich [6] and Kemp [7], and brief accounts of some general results may be found in O'Neill [8] and Beem and Ehrlich [9]. More recently, Deszcz et al [10] looked into the symmetry properties of the Riemann tensor of these spacetimes.

In the present paper we provide an invariant characterization of warped spacetimes based mainly in the holonomy classification in Hall and Kay [2] and put forward a classification scheme for these spacetimes. This is done in section 2 . In section 3 we study the curvature structure, i.e. the allowed Petrov and Segre types of the Weyl and Ricci tensors in each case and give some specific results in particular cases.

Section 4 is devoted to the study of the isometry group that each class of warped spacetime may admit; discussing both the general form of the Killing vectors and the maximal dimension of the Lie algebra that they form. 
Finally, in section 5 we present some examples and make some further considerations.

\section{Invariant characterization of the warped product spacetimes}

As stated in the previous section, a warped product spacetime $(M, g)$ is such that the spacetime manifold $M$ is the product of two others $M=M_{1} \times M_{2}$, each one of which is endowed with a metric; $h_{1}$ and $h_{2}$ respectively (such that the $g$ defined below is of Lorentz signature); and the spacetime metric $g$ can be written as

$$
g=h_{1} \oplus e^{2 \theta} h_{2}
$$

$\theta$ being a smoth real function, called the 'warping function'

$$
\theta: M_{1} \rightarrow \mathbb{R} \text {. }
$$

Given a certain spacetime, the question now arises as to how can one decide whether it is warped or not, or in other words whether there exists a coordinate change that brings the metric into the form (1), subject to the restriction (2).

To answer this question, first note that (1) can be re-written as

$$
g=e^{2 \theta}\left(h_{1}^{\prime} \oplus h_{2}\right)=e^{2 \theta} \hat{g}
$$

where $h_{1}^{\prime} \equiv e^{-2 \theta} h_{1}$ is a metric on $M_{1}$ by virtue of (2); so a warped spacetime $(M, g)$ may be thought of as conformal to a locally decomposable one $[1,2]$ say $(M, \hat{g})$; the conformal factor being a function of the coordinates of just one of the submanifolds whose product defines $M$. Next, let us give a brief summary of the results on locally decomposable spacetimes; which will be of use later on.

The spacetime $(M, \hat{g})$ will be locally decomposable if its holonomy group is nondegenerately reducible $[1,2]$, i.e. its holonomy type is $R_{2}, R_{3}, R_{4}, R_{6}, R_{3}, R_{10}$ or $R_{13}$ (see [11] for a definition of $R_{N}$ ); and globally decomposable if and only if $M$ is simply connected.

One then has the following possibilities [2] (see also [12,13]).

(i) The spacetime $(M, \hat{g})$ is $1+3$ metrically decomposable, i.e. it admits a global, non-null, convariantly constant, nowhere zero vector field. $(M, \hat{g})$ is then said to be $1+3$ spacelike or $1+3$ timelike depending on the nature of the three-dimensional submanifold orthogonal to the covariantly constant vector field. This corresponds to the holonomy types being $R_{13}$ (case $1+3$ spacelike) or $R_{3}, R_{6}$ or $R_{10}$ (case $1+3$ timelike). The line element associated with the metric $\hat{g}$ takes then the form

$$
\mathrm{d} \hat{s}^{2}=\epsilon \mathrm{d} u^{2}+h_{\alpha \beta}\left(x^{\gamma}\right) \mathrm{d} x^{\alpha} \mathrm{d} x^{\beta} \quad \epsilon= \pm 1 .
$$

If there exists another non-null, covariantly constant, nowhere zero vector field, then $(M, \hat{g})$ decomposes still further and it is then referred to as being $1+1+2$ spacelike or $1+1+2$ timelike in an obvious notation (holonomy types $R_{4}$ and $R_{2}$ respectively).

(ii) The spacetime $(M, \hat{g})$ is $2+2$ locally decomposable, i.e. no global, covariantly constant, nowhere zero vector field exists but two global, linearly independent recurrent null vector fields are admitted. The latter is equivalent to $(M, \hat{g})$ admitting two global, linearly independent covariantly constant tensor fields of rank 2 , such that

$$
\hat{g}_{a b}=\hat{P}_{a b}+\hat{Q}_{a b}
$$


that is $\hat{P}_{a b}$ and $\hat{Q}_{a b}$ are two-dimensional metrics acting on mutually orthogonal twodimensional surfaces; satisfying -as tensor fields on $\mathrm{M}$ -

$$
\hat{P}_{a b ; c}=\hat{Q}_{a b ; c}=0 \text {. }
$$

One can then choose coordinates in the two mutually orthogonal surfaces, say $\left\{x^{A}\right\}$, $A=1,2$ for the surface with metric $\hat{P}_{a b}\left(M_{1}\right.$ for instance) and $\left\{x^{\alpha}\right\}, \alpha=3,4$ for the surface with metric $\hat{Q}_{a b}\left(M_{2}\right)$; such that the line element can be (locally) written as

$$
\mathrm{d} \hat{s}^{2}=\hat{\imath}_{A B}\left(x^{D}\right) \mathrm{d} x^{A} \mathrm{~d} x^{B}+\hat{\jmath}_{\alpha \beta}\left(x^{\gamma}\right) \mathrm{d} x^{\alpha} \mathrm{d} x^{\beta} .
$$

The holonomy type in this case is $R_{7}$.

Returning to $(M, g)$ (the warped spacetime conformally related to $(M, \hat{g})$ via (3)), it appears natural to consider the following classes of warped spacetimes.

Class $A$. $(M, g)$ is conformally related to a $1+3$ locally decomposable spacetime $(M, \hat{g}) .\left(M=M_{1} \times M_{2}\right.$ and $\left.\hat{g}=h_{1}^{\prime} \oplus h_{2}\right)$.

Two different subclasses are to be distinguished here, depending on the respective dimensions of $M_{1}$ and $M_{2}$ :

(i) $\mathrm{A}_{1}: \operatorname{dim} M_{1}=1$ and $\operatorname{dim} M_{2}=3$. The line element associated with $g$ can therefore be written as

$$
\mathrm{d} s^{2}=\epsilon \mathrm{d} u^{2}+\mathrm{e}^{2 \theta(u)} h_{\alpha \beta}\left(x^{\gamma}\right) \mathrm{d} x^{\alpha} \mathrm{d} x^{\beta} \quad \epsilon= \pm 1 \quad \alpha, \beta, \ldots=1,2,3
$$

where $\{u\}$ is the local coordinate chart in $M_{1}$ and $\left\{x^{\gamma}\right\}_{\gamma=1,2,3}$ the one in $M_{2}$; and $h_{1}$ and $h_{2}$ read, in their respective charts: $h_{1}=\epsilon \mathrm{d} u \otimes \mathrm{d} u ; h_{2}=h_{\alpha \beta}\left(x^{\gamma}\right) \mathrm{d} x^{\alpha} \otimes \mathrm{d} x^{\beta}$.

The case $\epsilon=+1(-1)$ corresponds to $M_{1}$ being Riemannian (Lorentzian) and consequently $M_{2}$ is Lorentzian (Riemannian).

Since $h_{\alpha \beta}\left(x^{\gamma}\right)$ is a three-dimensional metric on $M_{2}$, it is always possible to cast it in diagonal form (irrespectively of its signature); and therefore in most cases we shall write the line element (8) as

$\mathrm{d} s^{2}=\epsilon \mathrm{d} u^{2}+\mathrm{e}^{2 \theta(u)}\left(-\epsilon \mathrm{e}^{2 A(v, x, y)} \mathrm{d} v^{2}+\mathrm{e}^{2 B(v, x, y)} \mathrm{d} x^{2}+\mathrm{e}^{2 C(v, x, y)} \mathrm{d} y^{2}\right)$.

Henceforth, we shall refer to (9) as the canonical form of the metric for warped spacetimes of the class $A_{1}$. Notice that all Robertson-Walker spacetimes belong to this class. form

(ii) $\mathrm{A}_{2}: \operatorname{dim} M_{1}=3, \operatorname{dim} M_{2}=1$. The associated line element then takes the

$$
\mathrm{d} s^{2}=\epsilon e^{2 \theta\left(x^{\gamma}\right)} \mathrm{d} u^{2}+h_{\alpha \beta}\left(x^{\gamma}\right) \mathrm{d} x^{\alpha} \mathrm{d} x^{\beta} \quad \epsilon= \pm 1 \quad \alpha, \beta, \ldots=1,2,3 .
$$

Now $\left\{x^{\gamma}\right\}_{\gamma=1,2,3}$ is the local coordinate chart in $M_{1}$ and $\{u\}$ the one in $M_{2}$; whilst $h_{1}$ and $h_{2}$ are written, in these charts, as $h_{1}=h_{\alpha \beta}\left(x^{\gamma}\right) \mathrm{d} x^{\alpha} \otimes \mathrm{d} x^{\beta}$ and $h_{2}=\epsilon \mathrm{d} u \otimes \mathrm{d} u$.

The same remarks as in the previous case, regarding signature and diagonability of $h_{\alpha \beta}$, also apply here. 
Class $B .(M, g)$ is conformally related to a $2+2$ locally decomposable spacetime $(M, \hat{g}), M=M_{1} \times M_{2}$ and $\hat{g}=h_{1}^{\prime} \oplus h_{2}$ with $\operatorname{dim} M_{1}=\operatorname{dim} M_{2}=2$. The line element of $(M, g)$ can then be written as

$$
\begin{gathered}
\mathrm{d} s^{2}=g_{A B}\left(x^{C}\right) \mathrm{d} x^{A} \mathrm{~d} x^{B}+\mathrm{e}^{2 \theta\left(x^{C}\right)} g_{\alpha \beta}\left(x^{\gamma}\right) \mathrm{d} x^{\alpha} \mathrm{d} x^{\beta} \quad \alpha, \beta, \ldots=3,4 \\
A, B, \ldots=1,2
\end{gathered}
$$

where $\left\{x^{A}\right\}_{A=1,2}$ and $\left\{x^{\alpha}\right\}_{\alpha=3,4}$ designate the local coordinate charts in $M_{1}$ and $M_{2}$ respectively, and $h_{1}=g_{A B}\left(x^{C}\right) \mathrm{d} x^{A} \otimes \mathrm{d} x^{B}, h_{2}=g_{\alpha \beta}\left(x^{\gamma}\right) \mathrm{d} x^{\alpha} \otimes \mathrm{d} x^{\beta}$ are their respective metrics expressed in the chosen coordinate charts.

Again the same comments about the nature (Riemann or Lorentz) of the submanifolds $M_{1}$ and $M_{2}$ apply here. Furthermore, since a two-dimensional space or spacetime is always conformally fiat, one can always write (11a) as

$$
\mathrm{d} s^{2}=\mathrm{e}^{2 A\left(x^{D}\right)}\left(\epsilon \mathrm{d} u^{2}+\mathrm{d} x^{2}\right)+\mathrm{e}^{2 \theta\left(x^{C}\right)} \mathrm{e}^{2 B\left(x^{\gamma}\right)}\left(-\epsilon \mathrm{d} v^{2}+\mathrm{d} y^{2}\right)
$$

and again $(11 b)$ will be referred to as the canonical form for warped spacetimes of the class B.

Notice that Schwarzschild, Vaidya, Reissner-Nordstron and, in general, all the (simply connected) spacetimes which admit a three-dimensional group of isometries acting transitively on non-null two-dimensional orbits, belong to this class (this includes all spherically symmetric solutions). This is due to a theorem by Schmidt [17] (see also [14]) stating that if a group $G_{r}$ of motions of $r=\frac{1}{2} d(d+1)$ parameters has orbits of dimension $d(d>1)$, then the orbits admit orthogonal surfaces.

One should notice that there are spacetimes that belong to both classes A and B, for example, spacetimes whose metric are of the form:

$$
\mathrm{d} s^{2}=\epsilon \mathrm{d} u^{2}+\mathrm{e}^{2 \theta(u)}\left(-\epsilon \mathrm{d} v^{2}+\mathrm{e}^{2 C(y, z)}\left(\mathrm{d} y^{2}+\mathrm{d} z^{2}\right)\right)
$$

and many others.

We shall designate this class of spacetimes as class $C$, i.e. $C=A \cap B$; but they will not be of much concern to us, since they can be regarded as special cases of either of the two previously defined classes.

In order to characterize each one of the classes $A_{1}, A_{2}, B$ of warped spacetimes invariantly, we recall the special properties (preferred vector or tensor fields) that the conformally related, locally decomposable spacetime has and look into what they imply in the warped one. Thus, if we re-write the line element (8) (i.e. class A spacetimes) as

$$
\mathrm{d} s^{2}=\mathrm{e}^{2 \theta(u)}\left\{\epsilon \mathrm{e}^{-2 \theta(u)} \mathrm{d} u^{2}+h_{\alpha \beta}\left(x^{\gamma}\right) \mathrm{d} x^{\gamma} \mathrm{d} x^{\beta}\right\}=\mathrm{e}^{2 \theta} \mathrm{d} \hat{s}^{2}
$$

and re-scale the coordinate $u$ to a new coordinate $u^{\prime}\left(\mathrm{d} u^{\prime}=\mathrm{e}^{-\theta} \mathrm{d} u\right)$, so as to have

$$
\mathrm{d} \hat{s}^{2}=\epsilon \mathrm{d} u^{\prime 2}+h_{\alpha \beta}\left(x^{\gamma}\right) \mathrm{d} x^{\alpha} \mathrm{d} x^{\beta}
$$

the vector field $\hat{u} \equiv \partial_{u}$, becomes a non-null, nowhere zero covariantly constant vector field in $(M, \hat{g})$ (and therefore, a Killing vector in this spacetime). It can immediately 
be seen that this vector field is now a (proper) conformal Killing vector (CKV) in $(M, g)$. If we now consider

$$
u^{a}=\mathrm{e}^{-\theta} \hat{u}^{a}
$$

i.e. unit vector (in $(M, g)$ ) parallel to the CKV $\hat{u}^{a}$; it is easy to see that its associated shear $\left(\sigma_{a b}\right)$, rotation $\left(\omega_{a b}\right)$ and acceleration $\left(\dot{u}_{a}\right)$ are all zero, and that its expansion $\Theta(\Theta \neq 0)$ is only a function of $u^{\prime}$ (equivalently of $u$ ); i.e.

$$
\begin{aligned}
& \sigma_{a b}=\omega_{a b}=\dot{u}_{a}=0 \\
& \Theta \neq 0 \quad \Theta_{, c} h_{a}^{c}=0
\end{aligned}
$$

where $h_{a b} \equiv g_{a b}-\epsilon u_{a} u_{b}$ is the orthogonal projector to $u^{a}$. (See Kramer et al [14] for the definitons of $\sigma_{a b}, \omega_{a b}, \dot{u}_{a}, \theta$ in the case $u^{a}$ timelike $(\epsilon=-1)$. The proof for $u^{a}$ being spacelike follows along the same lines; for the definition of shear, rotation, acceleration and expansion in this case see [15].)

Conversely, if a spacetime admits a global, non-null, nowhere zero unit vector field which is geodesic, shearfree, hypersurface orthogonal, and such that the gradient of its expansion $\Theta$ is parallel to it, then a coordinate system exists [16] in which the metric takes the form (8) with

$$
\Theta \equiv 3 u^{b} \theta_{, b}=3 \mathcal{L}_{u} \theta
$$

We have thus proven the following proposition.

Propasition 1. The necessary and sufficient condition for a spacetime $(M, g)$ be warped of class $A_{1}$ is that a global, non-null, nowhere zero unit vector field exists that is geodesic, hypersurface orthogonal, shearfree and such that its expansion $\Theta$ satisfies $\Theta_{, c} h_{a}^{c}=0$.

A similar result holds for warped spacetimes of the class $A_{2}$, although in this case the preferred vector field is no longer geodesic, and it turns out that it must be non-expanding; so we can state the following proposition.

Proposition 2. The necessary and sufficient condition that a spacetime $(M, g)$ be warped of class $A_{2}$ is that a global, non-null, nowhere zero unit vector field exists that is hypersurface orthogonal, shear-free, non-expanding and such that its acceleration is a gradient (it turns out that $\left.\dot{u}_{a}=\left(\mathrm{e}^{\theta}\right)_{, a}\right)$.

The proof is almost identical to the one sketched earlier.

These two propositions can easily be seen to be equivalent to the following two theorems.

Theorem 1. Let $(M, g)$ be a spacetime. Then the following conditions are equivalent.

(a) $(M, g)$ is conformally equivalent to a $1+3$ locally decomposable spacetime.

(b) There exists a nowhere zero, nowhere null, hypersurface orthogonal conformal Killing vector $\hat{u}$ in $M$ such that if $\theta \equiv \sqrt{\epsilon g(\hat{u}, \hat{u})}$, then the vector field $u=\mathrm{e}^{-\theta} \hat{u}$ is shearfree and $\dot{u}_{a}=-\epsilon \theta_{, a}+\theta_{m} u^{m} u_{a}, \Theta=3 u^{m} \theta_{, m}$. 
Theorem 2. Let $(M, g)$ be a spacetime conformally equivalent to a $1+3$ locally decomposable spacetime; and let $u$ be the vector field whose existence is guaranteed by theorem 1 . Then

(a) $(M, g)$ is of class $A_{1}$ if and only if $\dot{u}_{a}=0$;

(b) $(M, g)$ is of class $A_{2}$ if and only if $\Theta=0$.

The existence of further non-null vector fields in $M$ with the same properties as $u$ would then imply that in fact $(M, g)$ belongs to class C. Class B warped product spacetimes cannot be characterized in such a neat way as spacetimes of class $A$. Nevertheless, an invariant characterization is still possible and, as in the previous case, it follows from the existence of vector fields with special properties in the conformally related $2+2$ locally decomposable spacetime $(M, \hat{g})$. A $2+2$ locally decomposable spacetime always admits two null recurrent vector fields, $\hat{l}^{a}$ and $\hat{k}^{a}$, such that $\hat{l}^{a} \hat{k}_{a}=-1[13]$; and they can always be scaled in such a way that the recurrence vector is parallel to one of them, say $\mathfrak{l}^{a}$, i.e.

$$
\hat{l}_{a / b}=\alpha \hat{l}_{a} \hat{l}_{b} \quad \hat{k}_{a / b}=-\alpha \hat{k}_{a} \hat{l}_{b}
$$

where $\alpha$ is a smooth real function of the coordinates associated with the integrable distribution spanned by $\hat{l}^{a}$ and $\hat{k}^{a}$; and a stroke denotes the covariant derivative with respect to the connection associated to $\hat{g}$.

If one defines now vector fields $l^{a}, k^{a}$ in $(M, g)$ as $l^{a} \equiv \mathrm{e}^{-\theta} \hat{l}^{a}$ and $k^{a} \equiv \mathrm{e}^{-\theta} \hat{k}^{a}$ (associated 1-forms $l_{a}=\mathrm{e}^{\theta} \hat{l}_{a}, k_{a}=\mathrm{e}^{\theta} \hat{k}_{a}$ satisfying $l^{a} k_{a}=-1$ ) and computes their covariant derivatives in the connection associated with $g$, one gets (see [14] for the relationship between the connections associated with $g$ and $\hat{g}$ ):

$$
\begin{aligned}
& l_{a ; b}=\beta l_{a} l_{b}-\theta_{, a} l_{b}+g_{a b}\left(\theta_{, c} l^{c}\right) \\
& k_{a ; b}=-\beta k_{a} l_{b}-\theta_{, a} k_{b}+g_{a b}\left(\theta_{, c} k^{c}\right)
\end{aligned}
$$

where $\beta \equiv \alpha e^{-\theta}$

It is easy to see now from (20) and the expressions of the Newman-Penrose spin coefficients $\kappa, \sigma$ and $\omega$ (see, for instance, [14] p 78) specified to $l_{a}$ and $k_{a}$, that both $l^{a}$ and $k^{a}$ are geodesic (although non-affinely parametrized), shearfree and hypersurface orthogonal, and their respective expansions are given by

$$
\Theta_{l}=\theta_{, c} l^{c} \quad \Theta_{k}=\theta_{, c} k^{c} \text {. }
$$

Had we defined $L^{a} \equiv \mathrm{e}^{-2 \theta} \hat{l}^{a}$ and $K^{a} \equiv \hat{k}^{a}\left(L^{a} K_{a}=-1\right)$ their covariant derivatives would read

$$
\begin{aligned}
& L_{a ; b}=\alpha L_{a} L_{b}-\theta_{, a} L_{b}-\theta_{b} L_{a}+\left(\theta_{, c} L^{c}\right) g_{a b} \\
& K_{a ; b}=-\alpha K_{a} L_{b}+\theta_{, b} K_{a}-\theta_{, a} K_{b}+\left(\theta_{, c} K^{c}\right) g_{a b}
\end{aligned}
$$

and again $L^{a}$ and $K^{a}$ would turn out to be hypersurface orthogonal, shearfree and geodesic, but in this case $L^{a}$ is affinely parametrized. Their expansions are

$$
\Theta_{L}=\theta_{, c} L^{c} \quad \Theta_{K}=\theta_{, c} K^{c} .
$$

The expressions (20) and (21) or, alternatively, (22) and (23) lead to an invariant characterization of $2+2$ warped product spacetimes. Using one or the other is just a matter of choice. We can therefore state the following theorem. 
Theorem 3. Let $(M, g)$ be a spacetime. Then

(a) If $(M, g)$ is conformally equivalent to a $2+2$ locally decomposable spacetime $(M, \hat{g})$ with $g=e^{2 \theta} \hat{g}$ then there exist null geodesic, shearfree and hypersurface orthogonal vector fields $l^{a}, k^{a}$ (or alternatively $L^{a}, K^{a}$ ) on $M$ whose covariant derivatives are given by (20) and their expansions by (21) (respectively (22) and (23)).

(b) If there exists a function $\theta: M \rightarrow \mathbb{R}$ and null vector fields $l^{a}, k^{a}$ on $M$ satisfying $l^{a} k_{a}=-1$ (or null vector fields $L^{a}, K^{a}$ satisfying $L^{a} K_{a}=-1$ ) and their covariant derivatives are given by (20) (respectively (22)), then the spacetime is conformal to a $2+2$ locally decomposable spacetime $(M, \hat{g})$ where $\hat{g}=\mathrm{e}^{-2 \theta} g$.

Theorem 4. Let $(M, g)$ be a spacetime conformally equivalent to a $2+2$ locally decomposable spacetime $(M, \hat{g})$; and $\theta, l^{a}, k^{a}$ (or $\theta, L^{a}, K^{a}$ ) the function and vector fields whose existence is guaranteed by Theorem 3 . Let $h_{a b} \equiv 2 l_{(a} k_{b)}$. and $h_{a b}^{\perp} \equiv g_{a b}-h_{a b}\left(H_{a b} \equiv 2 L_{(a} K_{b)}, H_{a b}^{\perp} \equiv g_{a b}-H_{a b}\right)$. Then $(M, g)$ is a warped spacetime of class $\mathrm{B}$ if and only if either $h_{a b} \theta^{b}=0$ or $h_{a b}^{\perp} \theta^{b}=0$ (respectively $H_{a b} \theta^{b}=0$ or $H_{a b}^{\perp} \theta^{b}=0$ ).

Notice that in the first situation, i.e. $h_{a b} \theta^{b}=H_{a b} \theta^{b}=0$; the vector fields $l^{a}$ and $k^{a}\left(L^{a}\right.$ and $K^{a}$ ) are expansion-free. This case corresponds to $M_{1}$ being Riemannian, whilst the other possibility, namely $h_{a b}^{\frac{1}{b}} \theta^{b}=0$, takes place when $M_{1}$ is Lorentz.

We have now a complete characterization of warped product spacetimes.

\section{The curvature structure}

In this section we shall study the possibilities for the Petrov and Segre types of the Weyl and Ricci tensors, respectively, for the different classes of warped product spacetimes.

Since every warped product spacetime is conformally related to a locally decomposable spacetime and conformal scaling of the metric does not affect the Weyl tensor, the allowed Petrov types for a given class of warped spacetimes will be exactly those allowed for the type of locally decomposable spacetime that this class is related to and one can simply read this information from the table in [2].

Thus, class $A$ warped spacetimes are conformal to $1+3$ locally decomposable spacetimes; i.e. holonomy types $R_{3}, R_{6}$ or $R_{10}$ in the case $1+3$ timelike (the covariantly constant vector field in the decomposable spacetime is spacelike); and $R_{13}$ when it is $1+3$ spacelike (the covariantly constant vector field is timelike). In the first case $(1+3$ timelike) there are no restrictions on the Petrov type of the Weyl tensor, while in the second case $(1+3$ spacelike) it can only be $I, D$ or 0 . Class B warped spacetimes are conformal to $2+2$ locally decomposable spacetimes whose holonomy type is $R_{7}$; therefore [2] their Petrov type can only be D or 0 .

Unfortunately, conformal scaling of the metric does change the Ricci tensor in general, and therefore looking into the conformally related locally decomposable spacetime is of little or no use in order to work out the algebraic structure of the Ricci tensor in the warped spacetime. Nevertheless, a few conclusions can still be reached. 
Proposition 3. Let $(M, g)$ be a warped product spacetime of class $\mathrm{A}$; then, the (nonnull) vector field spanning the one-dimensional submanifold ( $M_{1}$ for subclass $\mathrm{A}_{1}$ and $M_{2}$ for subclass $\mathrm{A}_{2}$ ) is a Ricci eigenvector.

Recalling that $g=\mathrm{e}^{2 \theta} \hat{g}$, where $(M, \hat{g})$ is a $1+3$ decomposable spacetime, and that the unit vector spanning the one-dimensional submanifold in $(M, \hat{g})$, say $\hat{u}^{a}$, is covariantly constant with respect to the connection associated with $\hat{g}$; i.e. $\hat{u}_{a / b}=0$; one has:

(a) Subclass $A_{1}: \theta=\theta(u)$. Define now the unit vector field $u^{a}$ in $(M, g)$ as

$$
u^{a}=\mathrm{e}^{-\theta} \hat{u}^{a}
$$

i.e. $u_{a}=\mathrm{e}^{\theta} \hat{u}_{a}$. One has $\theta_{, c}=\dot{\theta} \hat{u}_{c}=\dot{\theta} \mathrm{e}^{-\theta} u_{c}$, where a dot indicates differentiation with respect to the coordinate $u$. Evaluating now the Ricci identity for $u_{a}$ in $(M, g)$, it follows that

$$
u_{a ; b c]}=-\frac{1}{2} \mathrm{e}^{-2 \theta} \ddot{\theta}\left(g_{a b} u_{c}-g_{a c} u_{b}\right)=\frac{1}{2} u_{d} R_{a b c}^{d} .
$$

Contracting now with $g^{a b}$ one gets

$$
R^{d}{ }_{c} u_{d}=3 \ddot{\theta} \mathrm{e}^{-2 \theta} u_{c} \text {. }
$$

(b) Subclass $\mathrm{A}_{2}: \theta=\theta\left(x^{\gamma}\right), \theta,{ }_{, u}=0$. Proceeding as before we get, in this case,

$$
R^{d}{ }_{c} u_{d}=-g^{a b}\left(\theta_{; a b}+\theta_{a} \theta_{b}\right) u_{c}
$$

which completes the proof of the proposition.

Notice that if the coordinate $u$ is timelike $\left(M_{1}\right.$ or $M_{2}$ of Lorentz type in the subclasses $A_{1}$ and $A_{2}$ respectively), the Ricci tensor is of the diagonal Segre type [18], since it admits a timelike eigenvector.

In the case of warped spacetimes of class $B$, there are no restrictions on the Segre type, all types being possible in general.

To close this section, we next give the expression for the components of the Riemann and Ricci tensors, which will be useful for further developments. In what follows we shall denote the coordinates in $\left(M_{1}, h_{1}\right)$ by $x^{A, B, C}, \ldots$ and those in $\left(M_{2}, h_{2}\right)$ by $x^{\alpha, \beta, \gamma} \cdots$, and the geometrical objects (connection, Riemann tensor, etc) defined on each one of them by means of an index 1 or 2 respectively. Also, we shall write $n$ for the dimension of $M_{2}(n=1,2$ or 3$)$.

We now have, for the Riemann tensor,

$$
\begin{aligned}
& R_{B C D}^{A}={ }^{1} R_{B C D}^{A} \\
& R_{\beta C D}^{A}=R_{B \gamma D}^{A}=R_{B \gamma \delta}^{A}=R_{\beta \gamma \delta}^{A}=0 \\
& R_{B C D}^{\alpha}=R_{\beta C D}^{\alpha}=R_{B \gamma \delta}^{\alpha}=R_{\beta C \delta}^{\alpha}=0 \\
& R_{\beta C \delta}^{A}=-\mathrm{e}^{\theta}\left(\mathrm{e}^{\theta}\right)_{; C}^{A} h_{\beta \delta} \\
& R_{B \gamma D}^{\alpha}=-\mathrm{e}^{-\theta}\left(\mathrm{e}^{\theta}\right)_{; B D} \delta_{\gamma}^{\alpha} \\
& R_{\beta \gamma \delta}^{\alpha}={ }^{2} R_{\beta \gamma \delta}^{\alpha}+\left(\theta_{E} \theta^{E}\right) \mathrm{e}^{2 \theta}\left({ }_{2}^{h}{ }_{\beta \gamma} \delta_{\delta}^{\alpha}-h_{\beta \delta} \delta_{\gamma}^{\alpha}\right) .
\end{aligned}
$$


From these expressions it can immediately be seen that the components of the Ricci tensor are

$$
\begin{aligned}
& R_{B D}={ }^{1} R_{B D}-n \mathrm{e}^{-\theta}\left(\mathrm{e}^{\theta}\right)_{; B D} \\
& R_{B \delta}=0 \\
& R_{\beta \delta}={ }^{2} R_{\beta \delta}+\left\{4(1-n) \mathrm{e}^{2 \theta}\left(\theta_{E} \theta^{E}\right)-\mathrm{e}^{\theta}\left(\mathrm{e}^{\theta}\right)_{; A}^{A}\right\}_{2} h_{\beta \delta}
\end{aligned}
$$

where all indices are raised and lowered using the metric of the spacetime $g$.

\section{Isometry groups of warped product spacetimes}

The purpose of this section is to carry out systematic research on the possible isometry groups that each class of warped product spacetimes may admit, giving to the furthest possible extent the expression for the Killing vectors in it. In order to do this, it will be useful to have the expressions for the covariant derivatives of vector fields and 1-forms defined on $(M, g)$ in terms of the covariant derivatives in $\left(M_{1}, h_{1}\right)$ and $\left(M_{2}, h_{2}\right)$ of their projections on these submanifolds. Following the same conventions as at the end of the last section, we shall have for the connection in $(M, g)$

$$
\begin{aligned}
& \Gamma_{B C}^{A}={ }^{1} \Gamma_{B C}^{A} \quad \Gamma_{B \gamma}^{A}=0 \quad \Gamma_{\beta \gamma}^{A}=-e^{2 \theta} \tilde{h}_{1}^{A D}{\underset{2}{h \gamma}}_{\beta \gamma} \theta, D \\
& \Gamma_{B C}^{\alpha}=0 \quad \Gamma_{B \gamma}^{\alpha}=\theta_{, B} \delta_{\gamma}^{\alpha} \quad \Gamma_{\beta \gamma}^{\alpha}={ }^{2} \Gamma_{\beta \gamma}^{\alpha}
\end{aligned}
$$

where $\tilde{h}_{1}^{A B}$ satisfies $\tilde{h}_{1}^{A B} h_{B C}=\delta_{C}^{A}$; i.e. is the inverse metric in $\left(M_{1}, h_{1}\right)$, and should not be confused with $h_{1}^{A B} \equiv g^{A c} g^{B d} h_{c d}$, namely the contravariant form of $h_{1}$ (considered as a tensor field on $(M, g)$ ) with indices raised with the full metric g. Although for $h_{1}, \tilde{h}_{1}^{A B}$ coincides with $h_{1}^{A B}$, this is not so for $h_{2}$; in fact one has

$$
\begin{aligned}
& h_{1}^{A B}=\bar{h}_{1}^{A B} \quad h_{1}^{A B}=h_{1}^{\alpha \beta}=0 \\
& h_{2}^{\alpha \beta}=\mathrm{e}^{-4 \theta} \tilde{h}_{2}^{\alpha \beta} \quad h_{2}^{\alpha B}=h_{2}^{A B}=0 \\
& g^{a b}=\tilde{h}_{1}^{a b}+\mathrm{e}^{-2 \theta} \tilde{h}_{2}^{a b} .
\end{aligned}
$$

Let $X$ be a vector field on $M$. Define its vertical and horizontal components, $\underset{v}{X}$ and $\underset{h}{X}$, as follows.

$$
\begin{aligned}
& \underset{v}{X^{a}} \equiv g^{a b} h_{b d} X^{d}=\underset{1}{h} \underset{d}{A} X^{d} \\
& \underset{h}{X^{a}} \equiv X^{a}-X_{v}^{a} .
\end{aligned}
$$

One readily sees that $\underset{v}{X} X^{A}=X^{A}, \underset{v}{X}{ }^{\alpha}=0$ and $\underset{h}{X}{ }^{\alpha}=X^{\alpha}, \underset{h}{X}{ }^{A}=0$. 
With these definitions, and taking into account (27), one has

$$
\begin{aligned}
& X_{; B}^{A}=\underset{v}{X} \underset{B}{A} \\
& X_{; \beta}^{A}=\underset{v, \beta}{X}-\mathrm{e}^{2 \theta} h_{1}^{A D} \theta_{, D}{ }_{2}^{h \gamma} \underset{h}{X}{ }^{\gamma} \\
& X_{; B}^{\alpha}=\underset{h}{X, B}{ }_{, B}^{\alpha}+\theta_{, B}{\underset{h}{\alpha}}^{\alpha} \\
& X_{; \beta}^{\alpha}=\theta_{, C} \underset{v}{X}{ }^{C} \delta_{\beta}^{\alpha}+\underset{h}{X ; \beta} \underset{\alpha}{\alpha} .
\end{aligned}
$$

For 1-forms $\omega$ on $M$; we also define vertical and horizontal components by first taking $\omega^{a}=g^{a b} \omega_{b}$, defining then $\underset{v}{\omega^{a}}$ and $\underset{h}{\omega^{a}}$ as before, and finally lowering indices with $g_{a b}$. We get

$$
\begin{aligned}
& \omega_{A ; B}=\omega_{v} ; ; B \\
& \omega_{A ; \beta}=\omega_{v} A, \beta-\theta_{, A} \omega_{h} \\
& \omega_{\alpha ; B}=w_{\nu, B}-\theta_{, B} \omega_{\tilde{h}}{ }_{\alpha} \\
& \omega_{\alpha ; \beta}=-\mathrm{e}^{2 \theta}{\underset{v}{D}}_{\theta, D} h_{2_{\alpha \beta}}+\omega_{h}{ }_{\alpha ; \beta}
\end{aligned}
$$

where

$$
\omega_{a}=g_{a b}\left(\omega_{v}^{b}+\omega_{h}^{b}\right)={\underset{v}{a}}_{a}+\mathrm{e}^{2 \theta}{\underset{h}{a}}_{a}
$$

Now let $X$ be a Killing vector (KV) of $(M, g)$; the Killing equations $X_{a ; b}+X_{b ; a}=0$ will now read

$$
\begin{aligned}
& \underset{v}{X}{ }_{A ; B}+\underset{v}{X}{ }_{B ; A}=0 \\
& \underset{v}{X} A, \beta+\mathrm{e}^{2 \theta} \underset{h}{X_{\beta, A}}=0 \\
& \underset{h}{X} \alpha ; \beta+\underset{h}{X}{ }_{\beta ; \alpha}+2 \theta_{, D} \underset{v}{X_{2}^{D}}{ }_{2} \alpha \beta=0
\end{aligned}
$$

or, equivalently, denoting by | and | the covariant derivatives induced in $M_{1}$ and $M_{2}$ by their respective metrics $h_{1}$ and $h_{2}$,

$$
\begin{aligned}
& X_{v}^{X \mid B}+\underset{v}{X}{ }_{B \mid A}=0 \\
& \underset{v}{X}{ }_{A, \beta}+\mathrm{e}^{2 \theta} \underset{h}{X}{ }_{\beta, A}=0 \\
& \underset{h}{X} \underset{\alpha \beta}{ }+\underset{h}{X}{ }_{\beta \mid \alpha}=-2 \underset{v}{X^{D} \theta_{, D}}{\underset{2}{\alpha \beta}}_{\alpha \beta} .
\end{aligned}
$$

In what is to follow we shall denote the Lie algebra of KVs on $(M, g)$ as $K(M, g)$, and $K\left(M_{1}, h_{1}\right)$ and $K\left(M_{2}, h_{2}\right)$ will denote, respectively, the Lie algebras of KVs on $\left(M_{1}, h_{1}\right)$ and $\left(M_{2}, h_{2}\right)$. 
We can now extract the following consequences from these equations.

(i) If $X \in K(M, g)$ with $\underset{h}{X}=0$, then $\underset{v}{X}$ is a smooth vector field of $\left(M_{1}\right)$ and $\underset{v}{X} \in K\left(M_{1}, h_{1}\right)$. Moreover $\underset{v}{X}{ }^{D} \theta_{, D}=0$.

(ii) If $\underset{v}{X} \in K\left(M_{1}, h_{1}\right)$ and $\underset{v}{X}{ }^{D} \theta_{, D}=0$, then $\underset{v}{X} \in K(M, g)$.

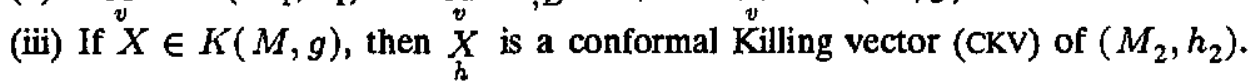
Furthermore, if $\underset{v}{X}=0$, then $\underset{h}{X}$ is a smooth vector field of $\left(M_{2}\right)$ and $\underset{h}{X} \in$ $K\left(M_{2}, h_{2}\right)$.

(iv) If $\underset{h}{X} \in K\left(M_{2}, h_{2}\right), \underset{h}{X} \in K(M, g)$.

Now fix $p_{2} \in M_{2}$ and consider the manifold $M_{1} \times\left\{p_{2}\right\} \simeq M_{1}$. Now (35) is a statement about KVs in $\left(M_{1}, h_{1}\right)$; suppose that $\operatorname{dim} K\left(M_{1}, h_{1}\right)=p \leqslant$ $n_{1}\left(n_{1}+1\right) / 2\left(n_{1} \equiv \operatorname{dim} M_{1}\right)$, and let $\left(V_{1}, \ldots, V_{p}\right)$ be a basis of $K\left(M_{1}, h_{1}\right)$; then there exist constants $\alpha^{I}\left(p_{2}\right), I=1 \ldots p$, such that $\underset{v}{X}=\sum_{I=1}^{p} \alpha^{I}\left(p_{2}\right) V_{I}$; i.e.

$$
\underset{v}{X}=\sum_{I=1}^{p} \alpha^{I} V_{I}
$$

where $\alpha^{I}=\alpha^{I}\left(x^{\alpha}\right)$ are functions depending on the variables $x^{\alpha}$ of $M_{2}$.

In the particular case $p=0$, one has $K(M, g) \simeq K\left(M_{2}, h_{2}\right)$ and it readily follows that $\operatorname{dim} K(M, g) \leqslant 6$ for class $\mathrm{A}_{1}, \operatorname{dim} K(M, g)=1$ for class $\mathrm{A}_{2}$ and $\operatorname{dim}$ $K(M, g) \leqslant 3$ for class $\mathrm{B}$.

Take now $p_{1} \in M_{1}$ and consider the manifold $\left\{p_{1}\right\} \times M_{2} \simeq M_{2}$. (37) is then a statement that $\underset{h}{X}$ is a $\mathrm{CKV}$ of $\left.\left(M_{2}, h_{2}\right) \underset{h}{X} \in C\left(M_{2}, h_{2}\right)\right)$. Proceeding as before, let us denote by $m$ the dimension of this Lie algebra, $m \equiv \operatorname{dim} C\left(M_{2}, h_{2}\right) \leqslant$ $\frac{1}{2}\left(n_{2}+1\right)\left(n_{2}+2\right)$ and by $\left(Y_{1} \ldots Y_{m}\right)$ a basis of it. Now, there exist constants $\lambda^{I}\left(p_{1}\right), I=1 \ldots m$, such that $\underset{h}{X}=\sum_{I=1}^{m} \lambda^{I}\left(p_{1}\right) Y_{I}$; i.e.

$$
\underset{h}{X}=\sum_{I=1}^{m} \lambda^{I} Y_{I}
$$

where $\lambda^{I}$ are now functions of the coordinates in $M_{1} ; \lambda^{I}=\lambda^{I}\left(x^{D}\right)$. Taking into account (38) and (39), it follows that $X \in K(M, g)$ must be of the form

$$
X=\sum_{J=1}^{p} \alpha^{J} V_{J}+\sum_{I=1}^{m} \lambda^{I} Y_{I}
$$

with $\alpha^{J}=\alpha^{J}\left(x^{\gamma}\right)$ and $\lambda^{I}=\lambda^{I}\left(x^{D}\right)$.

Notice that (40) is invariant under coordinate changes that preserve the warped product structure, namely

$$
x^{A^{\prime}}=F^{A}\left(x^{D}\right) \quad \text { and } \quad x^{\gamma^{\prime}}=f^{\gamma}\left(x^{\gamma}\right)
$$




\subsection{Warped spacetimes of class $A_{1}$}

Let $(M, g)$ be a class $\mathrm{A}_{1}$ warped product spacetime and assume its metric is given in the canonical form (9); namely

$$
\mathrm{d} s^{2}=\epsilon \mathrm{d} u^{2}+\mathrm{e}^{2 \theta(u)}\left(-\epsilon \mathrm{e}^{2 A(v, x, y)} \mathrm{d} v^{2}+\mathrm{e}^{2 B(v, x, y)} \mathrm{d} x^{2}+\mathrm{e}^{2 C(v, x, y)} \mathrm{d} y^{2}\right)
$$

and assume that $X \in K(M, g)$ is a $\mathrm{Kv}$. From (40) it follows that

$$
X^{a}=\epsilon \alpha(v, x, y) V^{a}+\sum_{I=1}^{m} \lambda^{I}(u) Y_{I}^{a}
$$

where $V$ is the vector spanning $K\left(M_{1}, h_{1}\right)$; in this case $V=\partial_{u}$ obviously; and $Y_{I}$ constitute a basis for $C\left(M_{2}, h_{2}\right)$ and therefore satisfies

$$
\begin{aligned}
& \left(\mathcal{L}_{Y_{I}} h\right)_{\alpha \beta}=2 \psi_{I}(v, x, y) \underset{2}{h_{\alpha \beta}} \\
& Y_{I}=b_{I}(v, x, y) \partial_{v}+c_{I}(v, x, y) \partial_{x}+d_{I}(v, x, y) \partial_{y} .
\end{aligned}
$$

The 1-form associated with $X$, given by (42), will be

$$
X_{a}=\alpha(v, x, y) V_{a}+\mathrm{e}^{2 \theta(u)} \sum_{I=1}^{m} \lambda^{I}(u) \tilde{Y}_{I a}
$$

where $\tilde{Y}_{I a}$ denote the 1 -forms in $\left(M_{2}, h_{2}\right)$ associated with the vector fields (44), i.e. with the indices lowered with $\frac{h}{2}$ :

$$
\tilde{Y}_{I}=-\epsilon \mathrm{e}^{2 A} b_{I} \mathrm{~d} v+\mathrm{e}^{2 B} c_{I} \mathrm{~d} x+\mathrm{e}^{2 C} \mathrm{~d}_{I} \mathrm{~d} y .
$$

The Killing equations (32)-(34) (or alternatively (35)-(37)) specialized to $X_{a}$ given by (45) now read

$$
\begin{aligned}
& \alpha_{, u}=0 \\
& \alpha_{, \beta}+\mathrm{e}^{2 \theta} \sum_{I} \dot{\lambda}^{I} \tilde{Y}_{I \beta}=0 \quad \beta=v, x, y \\
& \sum_{I} \lambda^{I}\left(\tilde{Y}_{I \alpha \mid \beta}+\tilde{Y}_{I \beta \mid \alpha}\right)=-\underset{2}{2 \alpha \dot{\theta}_{h} \alpha \beta}
\end{aligned}
$$

where a dot indicates differentiation with respect to $u$, as usual. Notice that (47) is trivially satisfied.

Differentiating now (48) with respect to $u$, and specifying the result for each index $\beta=v, x, y$; we obtain three equations; raising indices with $\bar{h}_{2}^{\alpha \beta}$, multiplying the equation corresponding to the (super)index $\alpha$ by $\partial_{\alpha}$ and adding the three of them up we obtain

$$
\mathrm{e}^{2 \theta} \sum_{I}\left(2 \dot{\theta} \dot{\lambda}^{I}+\ddot{\lambda}^{I}\right) Y_{I}=0 .
$$


Since this is a linear combination of linearly independent vectors (remember that $\left(Y_{I}\right)_{I=1}^{m}$ form a basis of $C\left(M_{2}, h_{2}\right)$ ) that equals zero, the coefficients must be all zero; i.e.

$$
2 \dot{\theta} \dot{\lambda}^{I}+\ddot{\lambda}^{I}=0
$$

Integration with respect to $u$ yields

$$
\lambda^{I}=p^{I} F(u)+q^{I} \quad \dot{F}(u)=\mathrm{e}^{-2 \theta}
$$

$p^{I}$ and $q^{I}$ being real constants.

Substituting these results in (49) we get

$$
\sum_{I=1}^{m}\left(p^{I} F+q^{I}\right)\left(Y_{I \alpha \mid \beta}+\left(Y_{I \beta|\alpha|}\right)=-2 \alpha \dot{\theta}_{2} h_{\alpha \beta} .\right.
$$

Now, since $K\left(M_{2}, h_{2}\right) \subset C\left(M_{2}, h_{2}\right)$ we can take the $\left(Y_{I}\right)_{I=1 \ldots m}$ (vectors of the basis of $C\left(M_{2}, h_{2}\right)$ ) in such a way that the first $p$ amongst them, i.e. $\left(Y_{L}\right)_{L=1 \ldots p} p \leqslant$ $m$, form a basis of $K\left(M_{2}, h_{2}\right)$ and the remaining $m-p \equiv q$ complete it to a basis of $C\left(M_{2}, h_{2}\right)$, thus being proper conformals (except, maybe, one homothety). This means that

$$
\begin{array}{ll}
Y_{L \alpha \mid \beta}+Y_{L \beta ! \alpha}=0 & L=1 \ldots p \\
Y_{I \alpha \mid \beta}+Y_{I \beta \mid \alpha}=2 \psi_{Y} & I=p+1 \ldots m .
\end{array}
$$

Equation (53) now reads

$$
\sum_{p+1 \leqslant I \leqslant m}\left(p^{I} F+q^{I}\right) \psi_{I}=-\alpha \dot{\theta}
$$

Differentiating with respect to $u$ and taking into account (52), it is easy to see that a necessary condition for (55) to hold is

$$
\begin{aligned}
& \ddot{\theta} \mathrm{e}^{2 \theta}= \pm k^{2} \\
& -\frac{1}{\alpha} \sum_{I} p^{I} \psi_{I}= \pm k^{2} .
\end{aligned}
$$

From (56) one can see that there are two possibilities:

(a) For all $k \in \mathbb{R}, \theta(u)$ is not a solution of (56). In this case, it follows that $p^{I}=q^{I}=0$ for $I=p+1 \ldots m$; and therefore

$$
\alpha=0 \text {. }
$$

A straightforward calculation shows that $p^{L}=0$ for $L=1 \ldots p$ also, and hence

$$
X=\sum_{L=1}^{p} q^{I} Y_{I} \quad q^{I} \in \mathbb{R}
$$


or equivalently $K(M, g) \cong K\left(M_{2}, h_{2}\right)$ and therefore $\operatorname{dim} K(M, g) \leqslant 6$.

(b) There exists $k \in \mathbb{R}$ such that $\theta$ is a solution of (56). In this case, the solutions of (56) can be given as

$$
\begin{aligned}
& \theta=\ln \cosh (k u) \quad \text { if the }+\operatorname{sign} \text { holds } \\
& \theta=\ln \sinh (k u) \quad \text { if the }-\operatorname{sign} \text { holds } \\
& \theta=a u+b \quad \text { if } k=0 .
\end{aligned}
$$

Plugging this back into (55), it follows that

$$
q^{I}=0 \quad I=p+1 \ldots m .
$$

The compatibility conditions (48) may now be written as

$$
\sum_{1 \leqslant L \leqslant p} p^{L} Y_{L}+\sum_{p+1 \leqslant I \leqslant m} k^{I}\left( \pm k^{2} Y_{I}+Z_{I}\right)=0
$$

where $p^{I}= \pm k^{2} k^{I}$ and

$$
Z_{I}=\epsilon \mathrm{e}^{-2 A} \psi_{I, v} \partial_{v}-\mathrm{e}^{-2 B} \psi_{I, x} \partial_{x}-\mathrm{e}^{-2 C} \psi_{I, y} \partial_{y} .
$$

Equation (62) sets restrictions on the maximum number of independent constants $p^{J}, J=1 \ldots m$; thus restricting $\operatorname{dim} K(M, g)$. Consider $E$, the real vector subspace spanned by $Y_{1} \ldots Y_{p}(\operatorname{dim} E=p), F$ the one spanned by $\pm k^{2} Y_{p+1}+$ $Z_{p+1}, \ldots, \pm k^{2} Y_{m}+Z_{m}(\operatorname{dim} F=q \leqslant m-p)$ and $G=F \cap E(\operatorname{dim} G=r \leqslant$ $\min (p, q)$ ); one can then come to the conclusion that

$$
\operatorname{dim} K(M, g)=m-q+r \leqslant m
$$

where $m=\operatorname{dim} C\left(M_{2}, h_{2}\right)$ is at most 10 (and if $m=10, h_{2}$ is conformally flat); $q=\operatorname{dim} F \leqslant m-p$ with $p=\operatorname{dim} K\left(M_{2}, h_{2}\right) \leqslant 6 ;$ and $r \leqslant \min (p, q)$. Recall that in this case $\theta(u)$ must take one of the expressions given by (60).

It is interesting to notice that the maximal dimension of a Lie algebra of Killing vectors, i.e. 10, can indeed be achieved in this case when $m=10\left(h_{2}\right.$ conformally flat) and $r=q$. The spacetime $(M, g)$ admitting $10 \mathrm{kVs}$, it must be of constant curvature and therefore one of the de-Sitter spacetimes; as it can be easily verified by direct computation in this framework (see next section).

\subsection{Warped spacetimes of class $A_{2}$}

We proceed as in the previous case by recalling the canonical form of the metric for this class of warped spacetimes

$$
\mathrm{d} s^{2}=\epsilon \mathrm{e}^{2 \theta(v, x, y)} \mathrm{d} u^{2}-\epsilon \mathrm{e}^{2 A(v, x, y)} \mathrm{d} v^{2}+\mathrm{e}^{2 B(v, x, y)} \mathrm{d} x^{2}+\mathrm{e}^{2 C(v, x, y)} \mathrm{d} y^{2} .
$$

Notice that these spacetimes always admit the Killing vector $\xi=\partial_{u}$. Expression (40) of a generic $\mathrm{KV}$ reads, in this case, as

$$
X=\sum_{I=1}^{m} \alpha^{I}(u) V_{I}^{a}+\lambda(v, x, y) Y^{a}
$$


where $\left(V_{I}\right)_{I=1 \ldots m}$ form a basis of $K\left(M_{1}, h_{1}\right)(m \leqslant 6)$ and $Y \in C\left(M_{2}, h_{2}\right)$ is a CKV of $\left(M_{2}, h_{2}\right)$ (notice that in this case, and since $\operatorname{dim} M_{2}=1$, the conformal algebra of $\left(M_{2}, h_{2}\right)$ is infinite dimensional; therefore we cannot speak about one single vector spanning $C\left(M_{2}, h_{2}\right)$; nevertheless (65) is still valid with $Y \in C\left(M_{2}, h_{2}\right)$ to be determined by the Killing equations)

$$
\begin{aligned}
& V_{I}=b_{I}(v, x, y) \partial_{v}+c_{I}(v, x, y) \partial_{x}+d_{I}(v, x, y) \partial_{y} \\
& Y=\epsilon f(u) \partial_{u}
\end{aligned}
$$

The 1 -form associated with $X$, given by $(65)$, will be

$$
X_{a}=\sum_{I=1}^{m} \alpha^{I}(u) V_{I a}+\mathrm{e}^{2 \theta(v, x, y)} \lambda(v, x, y) \bar{Y}_{a}
$$

where

$$
V_{I a}=g_{a b} V_{I}^{a}={\underset{1}{h a b}}_{I} V_{I}^{b} \quad \text { and } \quad \tilde{Y}_{a}=\underset{2}{h_{a b}} Y^{b}
$$

i.e. $\bar{Y}=f(u) \mathrm{d} u$. Now, the Killing equations (32)-(34) (or (35)-(37)) take the form:

$$
\begin{aligned}
& V_{I A \mid B}+V_{I B \mid A}=0 \quad I=1 \ldots m \\
& \sum_{I} \dot{\alpha}^{I} V_{I A}+f(u) \mathrm{e}^{2 \theta} \lambda_{, A}=0 \\
& \lambda\left(\bar{Y}_{\alpha \mid \beta}+\bar{Y}_{\beta \mid \alpha}\right)=-2 \sum_{I} \alpha^{I}\left(V_{I}^{D} \theta_{, D}\right){ }_{2}^{h_{\alpha \beta}} .
\end{aligned}
$$

Differentiating (70) with respect to $u$, it is easy to see that

$$
\alpha^{I}=p^{I} F(u)+q^{I} \quad \dot{F}(u)=f(u) \quad I=1 \ldots m
$$

where $p^{I}, q^{I}$ are real constants. Taking into account this information, and since $\bar{Y}_{\alpha \mid \beta}+\bar{Y}_{\beta \mid \alpha}=2 \dot{f}_{2}^{h}{ }_{\alpha \beta}$; it turns out that $(70)$ and $(71)$ can be re-written as

$$
\begin{aligned}
& \sum_{I} p^{I} V_{I A}+\mathrm{e}^{2 \theta} \lambda_{, A}=0 \\
& \lambda \dot{f}=-\sum_{I}\left(p^{I} F+q^{I}\right)\left(V_{I}^{D} \theta_{, D}\right) .
\end{aligned}
$$

Equation (73) can then be viewed as a compatibility condition, while (74) yields information on $f(u)$, since differentiating it with respect to $u$ we get

$$
\lambda \ddot{f}=-f \sum_{I} p^{I} V_{I}^{D} \theta_{, D}
$$


which trivially implies

$$
\begin{aligned}
& f / f=k \quad k=\text { constant } \\
& \sum_{I} p^{I}\left(V_{I}^{D} \theta_{, D}\right)=-k \lambda .
\end{aligned}
$$

Equation (75) can now be integrated for the different values of the constant $k$ giving

$$
\begin{array}{lcccrl}
f=\cosh (u) & \text { or } & & \sinh (u) & \text { for } & k=1 \\
f=\cos (u) & \text { or } & \sin (u) & \text { for } & k=-1 \\
f=k_{1} u+k_{2} & \text { for } & k=0 & & &
\end{array}
$$

where we have rescaled the coordinate $u$ in order to get these values for $k$.

Subsitituting back in (74) the different values of $f$ given by (77) and taking into account (76) it follows that

$$
\begin{aligned}
& \sum_{I} q^{I} V_{I}^{D} \theta_{, D}=0 \quad \text { and } \quad \sum_{I} p^{I} V_{I}^{D} \theta_{, D}=-k \lambda \quad \text { for } k \neq 0 \\
& \sum_{I} p^{I} V_{I}^{D} \theta_{, D}=0 \quad \text { and } \quad \sum_{I} q^{I} V_{I}^{D} \theta_{, D}=-k_{1} \lambda \quad \text { for } k=0 .
\end{aligned}
$$

Equation (78a) implies that $\eta \equiv \sum_{I} q^{I} V_{I} \in K\left(M_{1}, h_{1}\right)$, leaves $\theta$ invariant and is therefore a Killing vector of $(M, g)$, i.e. $\eta \in K(M, g)$. Similar remarks apply to $\xi^{\prime} \equiv \sum_{I} p^{I} V_{I}$ in the case $k=0$.

Now, the set of KVs (of $\left(M_{1}, h_{1}\right)$ ) that leave a given function invariant, in our case the warping function $\theta$, form a Lie subalgebra of $K\left(M_{1}, h_{1}\right)$ which we shall denote as $K_{\theta}$. It is a classical result (see for instance [19]) that given an $r$-parameter group of isometries $G_{r}$ acting on $s$-dimensional orbits in an $n$-dimensional manifold $V_{n}(s \leqslant n)$; there are $n-s$ functions on $V_{n}$ which are left invariant by $G_{r}$. In the present case and according to this result, we shall have $\operatorname{dim} K_{\theta}=p \leqslant 3(p \leqslant m)$; and if $p=3$ then the three KVs spanning $K_{\theta}$ must act on two-dimensional orbits. The general case, though, will be that of $K_{\theta}=\varnothing$. It will also be useful (when studying the case $k=0$ ) to consider the subset of $K\left(M_{1}, h_{1}\right)$ formed by those $\mathrm{KVs}$ (of $\left(M_{1}, h_{1}\right)$ ) $\eta_{s}, s=1 \ldots q$ satisfying $\mathcal{L}_{\eta_{s}} \theta=$ constant. It is easy to see that this set also forms a Lie algebra, namely $H_{\theta} \supseteq K_{\theta}$, such that given any two vectors in $H_{\theta}$ there always exists a linear combination of them that belongs to $K_{\theta}$; therefore $\operatorname{dim} H_{\theta}=q \leqslant p+1$. Thus, $\operatorname{dim} H_{\theta} \leqslant 4$; and if it is 4 then $H_{\theta}$ contains a three-dimensional subalgebra $K_{\theta}$ of KVs that leave $\theta$ invariant and that act on two-dimensional orbits.

Notice that $\lambda=$ constant implies, from (73), that $p^{I}=0 \quad I=1, \ldots, m$; which in turn implies $\lambda=0$ in the case $k \neq 0$ (from (76)) and $\sum_{I} q^{I} V_{I}^{D} \theta_{, D} \equiv \mathcal{L}_{\eta} \theta=$ constant, in the case $k=0$. The converse also holds, i.e. $p^{I}=0, I=1, \ldots, m$ implies $\lambda=0$ for $k \neq 0$, and $\lambda=$ constant and hence $\sum_{I} q^{I} V_{I} \equiv \eta \in H_{\theta}$ for $k=0$.

Given a warped spacetime of this class, it is easy to see that there is only one value of $k$ possible; i.e. one cannot have KVs corresponding to different values of $k$ in the same spacetime since othenwise they would not form a Lie algebra. We shall therefore analyse the problem for each value of $k$ separately. 
(a) Assume now $k=0$ and $\epsilon=1$ for the sake of simplicity, and consider two generic KVs of $(M, g)$; according to our previous developments they will read as

$$
\begin{aligned}
& X_{1}=f \lambda_{1} \partial_{u}+F \xi_{1}+\eta_{1} \\
& X_{2}=f \lambda_{2} \partial_{u}+F \xi_{2}+\eta_{2}
\end{aligned}
$$

where

$$
\begin{array}{ll}
\xi_{i} \equiv \sum_{I=1}^{m} p_{i}^{I} V_{I} \quad \eta_{i} \equiv \sum_{I=1}^{m} q_{i}^{I} V_{I} \quad i=1,2 \\
\lambda_{i, A}=-\mathrm{e}^{-2 \theta} \xi_{i A} & -k_{1} \lambda_{i}=\mathcal{L}_{\eta_{i}} \theta \quad i=1,2
\end{array}
$$

and $\xi_{i} \in K_{\theta}, i=1,2$ (and therefore $\xi_{i} \in K(M, g)$ ), namely

$$
\mathcal{L}_{\xi_{i}} \theta=0 \quad i=1,2 .
$$

Define $\dot{X}_{i} \equiv\left[\xi, X_{i}\right]$ and $\ddot{X}_{i} \boxminus\left[\xi, \dot{X}_{i}\right]$, which must also belong to $K(M, g)$, and consider now $\left[X_{i}, X_{j}\right] ;\left[X_{i}, X_{j}\right] ;\left[X_{i}, \ddot{X}_{j}\right] ;\left[\dot{X}_{i}, \dot{X}_{j}\right]$ and $\left[\ddot{X}_{i}, \ddot{X}_{j}\right]$ for $i, j=$ 1,2. Next demand the value of each one of these Lie brackets to be a $\mathrm{KV}$ of $(M, g)$. The results can then be summarized as follows (we do not reproduce the calculations involved here since they are rather lengthy, but on the other hand quite straightforward).

(i) $K_{\theta}=\varnothing \Rightarrow \lambda_{i}=$ constant and $X_{2}=c X_{1}, c=$ constant. There are two possibilities: either $H_{\theta}=\varnothing$ or $\operatorname{dim} H_{\theta}=1$. In the first case $\lambda=0$ and $\operatorname{dim} K(M, g)=1$ and it is spanned by $\xi=\partial_{u}$. In the second case $\lambda=\lambda_{0} \neq 0$ and $\operatorname{dim} K(M, g)=2$ and it is spanned by $\xi=\partial_{u}$ and $X=f \lambda_{0} \partial_{u}+\eta$ with $\eta \in H_{\theta}$ such that $\mathcal{L}_{\eta} \theta=-k_{1} \lambda_{0}$.

(ii) $K_{\theta} \neq \varnothing$. If the KV $\xi_{1}\left(\xi_{1} \in K_{\theta}\right)$ appearing in (79) is null, hypersurface orthogonal (because of (81)) and such that $\left[\xi_{1}, \eta_{1}\right]=a \xi_{1}(a=$ constant or zero); then $X_{1} \in K(M, g)$ and $X_{2}$, also given by (79), is necessarily of the form $X_{2}=b X_{1}+Z$ where $b=$ constant and $Z \in K(M, g)$ is such that

$$
Z=f \lambda_{0} \partial_{u}+\eta
$$

with $\lambda_{0}=$ constant and $\eta \in H_{\theta}$ satisfies $L_{\eta} \theta=-k_{1} \lambda_{0}$. Needless to say, if $H_{\theta}-K_{\theta}=\varnothing$ then $Z=0$ and therefore $K(M, g)$ is spanned by $\xi=\partial_{u}, X_{1}, \dot{X}_{1}$ and $K_{\theta}$; thus $\operatorname{dim} K(M, g)=p+3\left(p \equiv \operatorname{dim} K_{\theta}\right)$. If $H_{\theta}-K_{\theta} \neq \varnothing$ then every $X \in K(M, g)$ is equal to $c \xi\left(\xi=\partial_{u}, c=\right.$ constant), $b X_{1}+Z$ or else $d \dot{X}_{1}$ ( $d$ $=$ constant) (or a linear combination of them). Given now $X^{\prime}=b^{\prime} X_{1}+Z^{\prime}$ it is easy to see that $Z^{\prime}=s Z+\zeta$ with $s=$ constant and $\zeta \in K_{\theta}$; thus in this case $\operatorname{dim} K(M, g)=p+4$.

Notice that if no null, hypersurface orthogonal $\mathrm{KV} \xi$ in $K_{\theta}$ satisfying $[\xi, \eta]=a \xi$ for some $\eta \in K\left(M_{1}, h_{1}\right)$ exists, then $K(M, g)$ is spanned by $\xi=\partial_{u}, X=$ $f \lambda_{0} \partial_{u}+\eta\left(\eta \in H_{\theta}\right.$ such that $\left.\mathcal{L}_{\eta} \theta=k_{1} \lambda_{0}\right)$ and $K_{\theta}$; its dimension being $p+2$ if $H_{\theta}-K_{\theta} \neq \varnothing$ or $p+1$ if $H_{\theta}-K_{\theta}=\varnothing$. In particular, if $\left(M_{1}, h_{1}\right)$ is Riemannian $(\epsilon=-1)$ it cannot contain a null vector, and then the latter holds. 
(b) Assume $k=1$ and $\epsilon=1$ as in the previous case for the sake of simplicity. Take also two generic KVs of $(M, g)$ :

$$
\begin{aligned}
& X_{1}=\lambda_{1} \cosh (u) \partial_{u}+\sinh (u) \xi_{1}+\eta_{1} \\
& X_{2}=\lambda_{2} \cosh (u) \partial_{u}+\sinh (u) \xi_{2}+\eta_{2}
\end{aligned}
$$

where

$$
\begin{aligned}
& \xi_{i}=\sum_{I=1}^{m} p_{i}^{I} V_{I} \quad \eta_{i} \equiv \sum_{I=1}^{m} q_{i}^{I} V_{I} \quad i=1,2 \\
& \mathcal{L}_{\xi_{i}} \theta=-\lambda_{i} \quad \lambda_{i, A}=-e^{-2 \theta} \xi_{i A} \quad i=1,2
\end{aligned}
$$

and $\eta_{i} \in K_{\theta}$ (and therefore $\eta_{i} \in K(M, g)$ ), i.e.

$$
\mathcal{L}_{\eta_{i}} \theta=0 \quad i=1,2 .
$$

Since $\eta_{i} \in K(M, g)$ it follows that $X_{i}-\eta_{i} \in K(M, g)$ and therefore we can drop $\eta_{1}$ and $\eta_{2}$ from the expressions (84) of $X_{1}$ and $X_{2}$. Defining as before $\dot{X}_{i} \equiv\left[\xi, X_{i}\right]$ (now $\ddot{X}_{i} \equiv\left[\xi, \dot{X}_{i}\right]=X_{i}$ ), considering $\left[X_{i}, X_{j}\right] ;\left[X_{i}, \dot{X}_{j}\right]$ and $\left[\eta, X_{i}\right]$ for $\eta \in K_{\theta}$, and demanding the results of these Lie brackets to be KVs of $(M, g)$ it follows that

(i) $K_{\theta}=\varnothing$; then $K(M, g)$ is spanned by $X_{1}=\lambda_{1} \cosh (u) \partial_{u}+\sinh (u) \xi_{1}, \dot{X}_{1}$ and $\xi=\partial_{u}$, where $\xi_{1} \in K\left(M_{1}, h_{1}\right)$ and satisfies the two equations (86) (for $i=1$ ) plus the condition

$$
\lambda_{1}^{2}-\mathcal{L}_{\xi_{1}} \lambda_{1}=\text { constant }
$$

The dimension of $K(M, g)$ is therefore 3 if such a vector $\xi_{1}$ exists or 1 if does not exist; in which case $\xi=\partial_{u}$ is the only $\mathrm{KV}$ that $(M, g)$ admits.

(ii) $K_{\theta} \neq \varnothing$. The are now two different situations to be taken into account; namely

- There exists some $\eta \in K_{\theta}$ satisfying $\left[\eta, \xi_{1}\right]=c \xi_{1}(c=$ constant); then $K(M, g)$ is spanned by $X_{1}$ defined as in (i), $\dot{X}_{1}, \xi=\partial_{u}$ and all those $\eta \in K_{\theta}$ satisfying the condition above; $\operatorname{dim} K(M, g) \leqslant p+3$ or even $\operatorname{dim} K(M, g) \leqslant p+1$ if it does not exist any such $\xi_{1} \in K\left(M_{1}, h_{1}\right)$.

- There does not exist any $\eta \in K_{\theta}$ satisfying $\left[\eta, \xi_{1}\right]=c \xi_{1}$. Then, $\operatorname{dim} K(M, g)=p+1$ and $K(M, g)$ is then spanned by $\xi=\partial_{u}$ and $K_{\theta}$.

The remaining case, namely $k=-1$, can be treated in exactly the same way as the present one, arriving at conclusions similar to those just outlined.

\subsection{Warped spacetimes of class $B$}

In this case we cannot use the same technique as in the two previous cases, since $C\left(M_{2}, h_{2}\right)$ is now infinite-dimensional, and we cannot write an easy-to-handle, generic form for the CKVs in it, as we did for the class $A_{2}$ warped spacetimes. We must therefore tackle the problem directly; to this end recall the canonical form of the metric for spacetimes of class B (11b)

$$
\mathrm{d} s^{2}=\mathrm{e}^{2 A(u, x)}\left(\epsilon \mathrm{d} u^{2}+\mathrm{d} x^{2}\right)+\mathrm{e}^{2 \theta(u, x)} \mathrm{e}^{2 B(v, y)}\left(-\epsilon \mathrm{d} v^{2}+\mathrm{d} y^{2}\right)
$$


and let $X$ be a $\mathrm{KV}$ of $(M, g)$

$$
X=\alpha \partial_{u}+\beta \partial_{x}+\gamma \partial_{v}+\delta \partial_{y}
$$

where $\alpha, \beta, \ldots$ are functions of the four coordinates $u, x, v, y$. Killing's equations now read

$$
\begin{aligned}
& \alpha \partial_{u} A+\beta \partial_{x} A+\partial_{u} \alpha=0 \\
& \partial_{u} \beta+\epsilon \partial_{x} \alpha=0 \\
& \alpha \partial_{u} A+\beta \partial_{x} A+\partial_{x} \beta=0 \\
& -\mathrm{e}^{2(\theta+B)} \partial_{u} \gamma+\mathrm{e}^{2 A} \partial_{v} \alpha=0 \\
& \mathrm{e}^{2(\theta+B)} \partial_{u} \delta+\epsilon \mathrm{e}^{2 A} \partial_{y} \alpha=0 \\
& -\epsilon \mathrm{e}^{2(\theta+B)} \partial_{x} \gamma+\mathrm{e}^{2 A} \partial_{v} \beta=0 \\
& \mathrm{e}^{2(\theta+B)} \partial_{x} \delta+\mathrm{e}^{2 A} \partial_{y} \beta=0 \\
& \gamma \partial_{v} B+\delta \partial_{y} B+\partial_{v} \gamma=-\left(\alpha \partial_{u} \theta+\beta \partial_{x} \theta\right) \\
& \partial_{v} \delta-\epsilon \partial_{y} \gamma=0 \\
& \gamma \partial_{v} B+\delta \partial_{y} B+\partial_{y} \delta=-\left(\alpha \partial_{u} \theta+\beta \partial_{x} \theta\right) .
\end{aligned}
$$

Notice that (89)-(92) imply that for every pair of fixed values of $v$ and $y$, say

$$
\left(v_{0}, y_{0}\right), \underset{v}{X} \boxminus \alpha\left(u, x, v_{0}, y_{0}\right) \partial_{u}+\beta\left(u, x, v_{0}, y_{0}\right) \partial_{v}
$$

is a $\mathrm{KV}$ of $\left(M_{1}, h_{1}\right)$; i.e. $\underset{v}{X} \in K\left(M_{1}, h_{1}\right)$; thus, if $\left(V_{I}\right) I=1, \ldots, m \leqslant 3$ is a basis of $K\left(M_{1}, h_{1}\right)$ it follows from the general considerations at the beginning of this section that it must be

$$
\underset{v}{X}=\sum_{I=1}^{m} \alpha^{I}(v, y) V_{I} .
$$

On the other hand, (97)-(99) imply that for every pair of fixed values of $u$ and $x$, $\left(u_{0}, x_{0}\right)$ the vector $\underset{h}{X} \equiv \gamma\left(u_{0}, x_{0}, v, y\right) \partial_{v}+\delta\left(u_{0}, x_{0}, v, y\right) \partial_{y}$ is a CKV of $\left(M_{2}, h_{2}\right)$; although as we have already pointed out we cannot now write an expression for $\underset{h}{X}$ equivalent to (100), since $C\left(M_{2}, h_{2}\right)$ is infinite-dimensional and its members cannot be expressed in a concise form which could be useful for our purposes.

To proceed further, let us consider the Ricci tensor of $(M, g)$; then if $X \in$ $K(M, g)$ we must have

$$
\mathcal{L}_{X} \text { Ricci }=0 .
$$


Recall now the structure of Ricci (equations (25)):

$$
\begin{aligned}
& R_{B D}={ }^{1} R_{B D}-2 \mathrm{e}^{-\theta}\left(\mathrm{e}^{\theta}\right)_{; B D} \\
& R_{B \delta}=0 \\
& R_{\beta \delta}={ }^{2} R_{\beta \delta}+\lambda_{2}^{h_{\beta \delta}}
\end{aligned}
$$

where

$$
\lambda \equiv-\left\{4 \mathrm{e}^{2 \theta}\left(\theta^{E} \theta_{E}\right)+\mathrm{e}^{\theta}\left(\mathrm{e}^{\theta}\right)_{; A}^{A}\right\}
$$

On the other hand, since $\underset{h}{X} \in C\left(M_{2}, h_{2}\right)$ we must have (see for instance [20]):

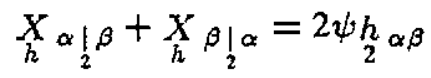

$$
\begin{aligned}
& \mathcal{L}_{\frac{X}{h}}{ }^{2} R_{\alpha \beta}=-2 \psi_{\alpha \mid \beta}-\left(h_{2}^{\gamma \delta} \psi_{\gamma \mid \delta}\right){ }_{2}^{h} h_{\alpha \beta}
\end{aligned}
$$

where $\psi=-\left(\alpha \partial_{u} \theta+\beta \partial_{x} \theta\right)$ as follows from (97) and (99). From all these considerations and after a long and tedious calculation, one can conclude that

(a) $\alpha^{I}(v, y)=$ constant, $I=1, \ldots, m$;

(b) $\underset{h}{X}=\gamma \partial_{v}+\delta \partial_{y}$ is a homothetic vector field of $\left(M_{2}, h_{2}\right)$ with homothetic factor $-\sum_{I} \alpha^{I} M_{I}$ where $M_{I} \equiv \mathcal{L}_{V_{I}} \theta, I=1, \ldots, m$.

There are now two subcases to be considered.

(i) $\left(M_{2}, h_{2}\right)$ has no proper homotheties. One must then have $\sum_{I} \alpha^{I} M_{I}=0$ and therefore

$$
\operatorname{dim} K(M, g)=l+\operatorname{dim} K\left(M_{2}, h_{2}\right)
$$

$l$ being the number of independent $\alpha^{I^{\prime}}$; which can be seen to be $0 \leqslant l \leqslant$ $m-p+q-1$, where $p \equiv \operatorname{dim} F, F$ being the vector space spanned by $Z_{I} \equiv\left(\partial_{u} M_{J}\right) \partial_{u}+\epsilon\left(\partial_{x} M_{I}\right) \partial_{x}, p \leqslant m \leqslant 3$ and $q=\operatorname{dim} K\left(M_{1}, h_{1}\right) \cap F$; since $\operatorname{dim} K\left(M_{1}, h_{1}\right)=m \leqslant 3$ it must be $q \leqslant \min (p, m)$; thus having $0 \leqslant q \leqslant p$, $0 \leqslant p \leqslant m$ and $0 \leqslant m \leqslant 3$.

(ii) $\left(M_{2}, h_{2}\right)$ admits a proper homothetic vector field. In this case one has

$$
\operatorname{dim} K(M, g)=l+\operatorname{dim} K\left(M_{2}, h_{2}\right)
$$

where $l$ (the number of independent $\alpha^{I^{\prime}}$ ) now satisfies $0 \leqslant l \leqslant m-p+q, p$ and $q$ defined as before.

Since $\operatorname{dim} K\left(M_{2}, h_{2}\right) \leqslant 3$ and $l \leqslant 3$ in both cases (i) and (ii), it follows that the maximal dimension of the Lie algebra of isometries for a warped spacetime of class $B$ is six. 


\section{Examples and concluding remarks}

As we have already pointed out in section 2 , all spacetimes admitting a threedimensional group of isometries acting transitively on non-null two-dimensional orbits (in particular all spherically symmetric solutions) are warped spacetimes of the class B; as follows from the theorem, due to Schmidt [17], that we quoted there. This theorem also allows us to conclude that all spacetimes which admit a six-parameter group of isometries acting on three-dimensional orbits are also warped spacetimes, belonging to the class $A_{1}$ in this case. These spacetimes include all Friedmann-RobertsonWalker cosmological models and many others (for a good survey see [14]). It is trivial to see that the converse does not hold.

It is also easy to see, as we mentioned in section 4.1, that the de-Sitter spacetimes are warped spacetimes of class $A_{1}$. Furthermore, they can be explicitly constructed by making use of the results derived in 4.1 .

On the other hand, important families of spacetimes such as the stationary axisymmetric solutions of the Einstein field equations are clearly non-warped, since the metric of a warped product spacetime can always be diagonalized. Thus, for instance, the Kerr solution and the Tomimatsu-Sato class of solutions are non-warped.

The problem of finding physically significant solutions belonging to the various classes of warped spacetimes is currently being studied by the authors, and the results will be presented elsewhere. Just to mention some results in this direction, one can show that the only perfect fluid solutions of class $\mathrm{A}_{1}$ with $\left(M_{1}, h_{1}\right)$ Lorentzian, are the Friedmann-Robertson-Walker cosmologies. One can also prove that this class (A $\mathrm{A}_{1}$, with $\left(M_{1}, h_{1}\right)$ Lorentzian) is emply for Einstein-Maxwell fields, both non-null and null.

The possible Lie algebra structures of $K(M, g)$ are also being investigated for the various classes presented here. A study of the conformal lie algebra of warped spacetimes is being carried out as an extension of the study of the Lie algebra of the isometries presented in section 4.

\section{Acknowledgments}

We would like to thank Professor L Mas and Dr C Bona, from the Universidad Illes Balears, and Dr G S Hall from the University of Aberdeen, for their valuable comments and suggestions. We are also grateful to the referees for their valuable suggestions and comments. One of the authors ( $\mathrm{J}$ da Costa) would like to thank the Departmento de Física of the Universidad de les Illes Balears for their kind hospitality during the preparation of this work. Partial support from CICYT/ Research grant PB 87-0583-C02-01 is also acknowledged.

\section{References}

[1] Wu H 1964 Illinois J. Math. 8291

[2] Hall G S and Kay W 1988 J. Math. Phys. 29420

[3] Allison D E 1985 Lorentzian warped products and statics space-times $P h D$ Thesis University of Missouri, Columbia

[4] Allison D E 1988 Gen. Rel Grav 20115

[S] Beem J K and Powell T G 1982 Tensor (NS) 3931 
[6] Beem J K and Ehrlich P E 1981 Gen. Rel Grav 13239

[7] Kemp M H 1981 Lorentzian warped products of a second type PhD Thesis University of Missouri, Kansas City

[8] O'Neill B 1983 Semi-Riemannian Geometry (New York: Academic)

[9] Beem J K and Ehrlich P E 1981 Global Lorentzian Geomety (New York: Marcel Dekker)

[10] Deszcz. R, Verstraelen L and Vrancken L 1991 Gen. Rel. Grav. 23671

[11] Schell J F 1961 J. Math. Phys: 2202

[12] Hall $G$ S and da Costa J 1988 J. Math Phys. 292465

[13] Hall G S $1991 \mathrm{~J}$. Moth. Phys. 32181

[14] Kramer D, Stephani H, Herit E and McCallum M A H 1980 Exact Solutions of Einstein's Field Equations (Cambridge: Cambridge University Press)

[15] Greenberg P J $1970 \mathrm{~J}$. Math AnaL AppL (USA) 30128

[16] Stephani H 1982 General Relativity. An Introduction to the Theory of the Gravitational Field (Cambridge: Cambridge University Press) p 162

[17] Schmidt B G 1967 Z. Narurf. a 221351

[18] Hall G S $1976 \mathrm{~J}$. Phys. A: Math. Gen. 9541

[19] Eisenhart L P 1961 Continuous Groups of Transformations (New York: Dover) p 62

[20] Hall G S $1990 \mathrm{~J}$. Math. Phys. 311198 